\title{
Prévalence De l’Obésité En Milieu Jeune En Côte d'Ivoire
}

\section{Adjoua Yeboua Florence Kouakou \\ Adama Kamagate \\ Angoué Paul Yapo}

Laboratoire de Physiologie animale, de Pharmacologie et de Phytothérapie,

UFR SN, Université Nangui Abrogoua, Abidjan, Côte d'Ivoire

doi: 10.19044/esj.2016.v13n3p241 URL:http://dx.doi.org/10.19044/esj.2016.v13n3p241

\begin{abstract}
Obesity is one of the diseases whose situation has become alarming in Côte d'Ivoire. As it is known, the young people represent the labor force of tomorrow. So we investigated the situation of obesity among youth. We took the case of Nangui Abrogoua University which is full of students from Ivory Coast, as well as students from countries in the sub region. Our study shows that the majority of students, about $74.01 \%$ had a normal weight. There is however $20.51 \%$ who was overweight, and $1.13 \%$ who was obese. Also overweight and abdominal obesity were more present in women than men. This low rate of obesity was explained by the fact that it is a more active population with a lower level of income. Our study highlighted that in addition to physical inactivity, obesity is also related to income level.
\end{abstract}

Keywords: Obesity, Diabetes, Body Mass Index, Waist circumference , Hypertension

\section{Résumé}

L’obésité est un fléau qui gagne du terrain en Côte d’Ivoire. Sachant que la population jeune d'aujourd'hui est la population adulte de demain, nous avons voulu voir qu'elle était la situation de l'obésité en milieu jeune. Nous avons pris le cas de l'Université Nangui Abrogoua qui regorge des étudiants Ivoiriens aussi bien que des étudiants venus des pays de la sous région. Notre étude montre que la majorité des étudiants, soit 74,01\% ont un poids normal. Il y a cependant $20,51 \%$ qui sont en surpoids et $1,13 \%$ qui sont obèses. Et la surcharge pondérale et l'obésité abdominale sont bien plus fréquentes chez les femmes que chez les hommes. Ce faible taux d'obésité s'explique par le fait que c'est une population bien active, et aussi qui a un 
niveau de revenu plus faible. Notre étude permet de mettre en lumière qu'en plus de la sédentarité, l’obésité est aussi liée au niveau de revenu.

Mots clés: Obésité, Diabète, Indice de masse Corporelle, Tour de taille, Hypertension Arterielle

\section{Introduction}

La surcharge pondérale constitue un des plus grands fléaux du XXIème siècle. La prévalence de l'obésité a triplé au cours de ces vingt dernières années et est considérée comme une véritable épidémie. Pour les plus de 15 ans, l'Organisation Mondiale de la Santé (OMS) estime le nombre de personnes en surpoids à 1,6 milliards, et obèses à 400 millions. Cette épidémie touche même les plus jeunes. En 2005, cette organisation a estimé à 20 millions le nombre d'enfants de moins de 5 ans en surpoids. Elle prévoit qu'en 2015, 2,3 milliards d'adultes seront en surpoids et 700 millions seront obèses (OMS, 2003).

L'obésité résulte d'un déséquilibre de la balance énergétique. Celle-ci représente la différence entre la totalité des dépenses énergétiques et la totalité des apports caloriques. Plusieurs facteurs interviennent simultanément dans le développement de cette maladie. Parmi les principaux facteurs, nous pouvons identifier : les comportements alimentaires, l'inactivité physique, les facteurs génétiques, l’origine ethnique, le niveau socio-économique, le tabac, les facteurs culturels, et la prise de certains médicaments.

De façon générale, l’obésité conduit à une diminution générale de la qualité de vie, à l'apparition de problèmes psychologiques, au dérèglement des cycles menstruels et à des complications lors des grossesses chez la femme. C’est un élément clé du syndrome métabolique. L'obésité engendre ainsi de nombreuses maladies telles: les maladies cardiovasculaires, le diabète de type 2, l'hypertension, l'apnée du sommeil, la dyslipidémie, et certains cancers. L'obésité pourrait participer à la diminution de l'espérance de vie des enfants qui naissent dans ces conditions, par rapport à celle de leurs parents (Olshansky, 2005). La prévention est encore le meilleur moyen de lutter contre l'obésité car une fois que la surcharge pondérale est en place, il est souvent très difficile de retrouver son poids idéal. Tout au long de la vie, il faut veiller à avoir une alimentation équilibrée et variée. La pratique d'une activité physique régulière permet de réguler les réserves d'énergie en augmentant l'utilisation des graisses. Il existe de très nombreux traitements contre l'obésité, notamment la prise en charge alimentaire, l'activité physique, les modifications de comportement, le traitement pharmacologique et la chirurgie gastrique. 
Afin de mieux comprendre comment se présente cette maladie en Côte d'Ivoire, nous allons de manière générale, faire une étude pour apporter des éléments de réponses sur le surpoids et l’obésité. Plus spécifiquement, nous avons étudié la prévalence de l’obésité des étudiants de l'Université Nangui Abrogoua (UNA) et identifier les facteurs à risques qui y sont associés.

\section{Matériel et Méthodes}

Cette étude a été menée à l’Université Nangui Abrogoua (UNA). Fondée en 1996, l'UNA était anciennement appelée Université AboboAdjamé (UAA). L’UNA est l'une des deux universités publiques d'Abidjan. L’UNA est située sur l'axe Abobo-Adjamé (autoroute d'Abobo). L'UNA est une université scientifique qui compte en son sein environ 5000 étudiants (4 449 en octobre 2012). L'université reçoit essentiellement des bacheliers de séries scientifiques en provenance de l'ensemble du territoire national, et aussi une minorité de la sous-région. L'Université Nangui Abrogoua comporte 4 unités de formations et de recherches ou UFR.

Dans cette étude, nous avons travaillé avec 177 sujets. La puissance statistique de l'échantillon a été estimée avec le logiciel $\mathrm{G}^{*}$ power 3.1.9.2 à un niveau de confiance d'au moins 95\% lorsque subdivisé en deux groupes, et avec une marge d'erreur inferieur à 0,05\%.

Cette étude a été réalisée sur des étudiants de l’UNA, une des universités publiques de la Côte d'Ivoire. Les participants à cette étude ont été choisis de manière aléatoire. La population de l'étude est estudiantine. Les étudiants étant la future population adulte ce qui fait d'eux une cible favorable pour des stratégies de sensibilisation et de prévention du surpoids et de l'obésité, des risques qu'elles peuvent engendrer surtout dans le cas du diabète et des dangers qui en découlent. Pour recueillir les données, une fiche d'enquête a été préalablement établie. Une campagne d'information a été menée auprès des étudiants concernés sur l'intérêt et les objectifs de ce travail. Les données ont été recueillies avec le consentement de chaque étudiant volontaire qui a voulu faire partir de l'échantillonnage. Pour l'aspect éthique, les données ont été recueillies dans le respect de la confidentialité et de l'anonymat. Ainsi :

(Soehnle) ;

- Le périmètre abdominal $(\mathrm{cm})$ a été pris en position debout à l'aide d'un mètre ruban. Il a été pris à la mi-distance entre la base thoracique et la crête iliaque (en regard du nombril) ;

- $\quad$ La tension artérielle également a été prise à l'aide d'un tensiomètre électronique à brassard (Spengler) ; 
- $\quad$ Et chaque étudiant a été interrogé sur ses antécédents familiaux en ce qui concerne le l'hypertension artérielle (HTA) et le diabète ; interrogé sur son statut marital, sur le revenu mensuel, sur le tabagisme.

Les informations recueillies sont :

- $\quad$ Des caractéristiques sociodémographiques (Genre, âge) ;

- Des caractéristiques anthropométriques (poids, taille, indice de masse corporelle (IMC), tour de taille (TT)) ;

- $\quad$ La tension artérielle ;

- Le statut financier ;

- Le statut marital ;

- Le tabagisme ;

- $\quad$ Les antécédents familiaux de HTA ou de diabète.

\section{Analyses statistiques}

Les données ont été saisies à l'aide du logiciel Excel et analysées à l'aide du logiciel statistica 7.1. La comparaison des moyennes a été effectuée par un test de Student. Les tests ont été jugés significatifs au niveau de confiance de $95 \%$ soit $\mathrm{p}<0,05$. La Puissance statistique de l'échantillon a été analysée avec le logiciel $G^{*}$ power 3.1.9.2. La taille de l’échantillon est jugée représentative au niveau de confiance de 95\%, avec une marge d'erreur de $0,05 \%$.

\section{Résultats}

\section{Comparaison du statut pondéral des hommes et femmes echantillonnés}

Sur les177 étudiants échantillonnés (Tableau 1), nous avons 50,85\% d'hommes et 49,15\% de femmes. $\pm 0,46$ ans.

Les hommes ont en moyenne $25,48 \pm 0,47$ ans et les femmes 24,99

Leur poids moyen des hommes est de $64,83 \pm 1,02 \mathrm{~kg}$ alors que celui des femmes est 58,24 $\pm 1,05 \mathrm{~kg}$.

La taille moyenne est de 1,73 $\pm 0,01 \mathrm{~m}$ chez l'homme et 1,63 $\pm 0,01 \mathrm{~m}$ chez la femme.

L'IMC des hommes est en moyenne de $21,55 \pm 0,29 \mathrm{~kg} / \mathrm{m}^{2}$ et celui des femmes est de $21,82 \pm 0,33 \mathrm{~kg} / \mathrm{m}^{2}$ Cette différence n'est pas significative $(p=0,269)$.

Le TT aussi n’est pas significativement important entre les femmes et les hommes $(p=0,314)$.

Aucun des hommes échantillonnés n’est marié alors que 5,75 \% des filles le sont.

96,67 \% des hommes ne fument, aucune femme échantillonnée ne fume. 
HTA.

Plus de la moitié des hommes et des femmes ont des parents avec

Tableau 1: Caractéristiques sociodémographiques des étudiants échantillonnés en fonction du Genre

\begin{tabular}{|c|c|c|c|}
\hline \multirow[t]{2}{*}{$\begin{array}{c}\text { Mesures } \\
\text { anthropométriques }\end{array}$} & Hommes & Femmes & $\mathrm{P}$ \\
\hline & $\mathrm{N}=90$ & $\mathrm{~N}=87$ & \\
\hline Age moyen & $25,48 \pm 0,47$ & $24,99 \pm 0,46$ & 0,228 \\
\hline Poids moyen & $64,83 \pm 1,02$ & $58,24 \pm 1,05$ & 0,0001 \\
\hline Taille moyenne & $1,73 \pm 0,01$ & $1,63 \pm 0,01$ & 0,0001 \\
\hline IMC moyen & $21,55 \pm 0,29$ & $21,82 \pm 0,33$ & 0,269 \\
\hline TT moyen & $75,08 \pm 0,91$ & $75,67 \pm 0,81$ & 0,314 \\
\hline TAS moyenne & $12,56 \pm 0,13$ & $11,36 \pm 0,11$ & 0,0001 \\
\hline TAD moyenne & $7,90 \pm 0,10$ & $7,39 \pm 0,09$ & 0,0001 \\
\hline \multicolumn{4}{|c|}{ Revenues/an } \\
\hline$<250000$ & $26(28,89 \%)$ & $36(41,38 \%)$ & \\
\hline$\geq 250000$ & $64(71,11 \%)$ & $51(58,62 \%)$ & \\
\hline \multicolumn{4}{|c|}{ Statut marital } \\
\hline Marié & 0 & $5(5,75 \%)$ & \\
\hline Célibataire & 90 (100\%) & $82(94,25 \%)$ & \\
\hline \multicolumn{4}{|c|}{ Tabagisme } \\
\hline Fumeur & $1(1,11 \%)$ & 0 & \\
\hline Non-fumeur & $87(96,67 \%)$ & 87 (100\%) & \\
\hline Passé de fumeur & $2(2,22 \%)$ & 0 & \\
\hline \multicolumn{4}{|c|}{ Antécédent familial } \\
\hline HTA famille & $48(53,33 \%)$ & $52(59,77 \%)$ & \\
\hline Diabète famille & $19(21,11 \%)$ & $20(22,99 \%)$ & \\
\hline HTA et diabète & $16(17,78 \%)$ & $19(21,84 \%)$ & \\
\hline
\end{tabular}

IMC : Indice de Masse Corporelle ; TT : Tour de Taille ; HTA : Hypertension Artérielle ;

TAD : Tension Artérielle Diastolique ; TAS : Tension Artérielle Systolique.

\section{Comparaison du statut pondéral des hommes et femmes selon la tranche d'age}

Selon le genre et la tranche d’âge, la prévalence de la surcharge pondérale augmente avec l'âge aussi bien chez les hommes que chez les femmes. Cependant, elle est plus élevée chez les femmes que chez les hommes (obésité abdominale : 26,44\% vs 1,11\%) (Tableau 2).

Tableau 2 : Statut pondéral des étudiants échantillonnés en fonction du genre et de la tranche d'âge

\begin{tabular}{|c|c|c|c|c|}
\hline Age (ans) & $\leq 24$ & $25-29$ & $\geq 30$ & Total \\
\hline \multicolumn{5}{|c|}{ HOMMES } \\
\hline \multicolumn{5}{|c|}{ P/r à l'IMC } \\
\hline Obèse & 0 & $2(6,67 \%)$ & 0 & $2(2,22 \%)$ \\
\hline Surpoids & $2(4,44 \%)$ & $1(3,33 \%)$ & $3(20 \%)$ & $10(11,11 \%)$ \\
\hline Normal & 37 (82,22\%) & $23(76,67 \%)$ & $10(66,67 \%)$ & $70(77,78 \%)$ \\
\hline Maigre & $6(13,33 \%)$ & $2(6,67 \%)$ & $2(13,33 \%)$ & $10(11,11 \%)$ \\
\hline
\end{tabular}




\begin{tabular}{|c|c|c|c|c|}
\hline \multicolumn{5}{|c|}{ P/r à TT } \\
\hline Obèse & 0 & $1(3,33 \%)$ & 0 & $1(1,11 \%)$ \\
\hline Non obèse & $45(100 \%)$ & $29(96,67 \%)$ & $15(100 \%)$ & $89(98,89 \%)$ \\
\hline \multicolumn{5}{|c|}{ FEMMES } \\
\hline \multicolumn{5}{|c|}{ P/r à l’IMC } \\
\hline Obèse & 0 & 0 & 0 & 0 \\
\hline Surpoids & $4(9,09 \%)$ & $6(19,35 \%)$ & $4(33,33 \%)$ & $14(16,09 \%)$ \\
\hline Normal & $33(75 \%)$ & $22(70,97 \%)$ & $6(50 \%)$ & $61(70,11 \%)$ \\
\hline Maigre & $7(15,91 \%)$ & $3(9,68 \%)$ & $2(16,67 \%)$ & $12(13,79 \%)$ \\
\hline \multicolumn{5}{|c|}{ P/r à TT } \\
\hline Obèse & $7(15,91 \%)$ & $12(29,27 \%)$ & $4(33,33 \%)$ & $23(26,44 \%)$ \\
\hline Non obèse & $37(84,09 \%)$ & $19(70,73 \%)$ & $8(66,67 \%)$ & $64(73,56 \%)$ \\
\hline
\end{tabular}

IMC : Indice de Masse Corporelle ; TT : Tour de Taille.

\section{Analyse du statut pondérale de l'ensemble des étudiants selon la trance d'age}

Chez l'ensemble des deux genres confondus, la tendance commune ne change pas. Mais la majorité des étudiants ont un poids normal aussi bien par rapport à l'IMC que par rapport au TT (Tableau 3).

Tableau 3 : Statut pondéral de l'ensemble des étudiants, les deux genres confondus en fonction de la tranche d'âge

\begin{tabular}{|c|c|c|c|c|}
\hline Age (ans) & $\leq 24$ & $25-29$ & $\geq 30$ & Total \\
\hline \multicolumn{5}{|c|}{ P/r à l'IMC } \\
\hline Obèse & 0 & $2(3,28 \%)$ & 0 & $2(1,13 \%)$ \\
\hline Surpoids & $6(6,74 \%)$ & $11(14,75 \%)$ & $7(25,93 \%)$ & $24(20,51 \%)$ \\
\hline Normal & $70(78,65 \%)$ & $45(73,77 \%)$ & $16(59,26 \%)$ & $131(74,01 \%)$ \\
\hline Maigre & $13(14,61 \%)$ & $5(16,13 \%)$ & $4(14,81 \%)$ & $22(12,43 \%)$ \\
\hline \multicolumn{5}{|c|}{$\mathrm{P} / \mathrm{r}$ à TT } \\
\hline Obèse & $7(7,87 \%)$ & $13(21,31 \%)$ & $4(14,81 \%)$ & $24(13,56 \%)$ \\
\hline Non obèse & $82(92,13 \%)$ & $48(78,69 \%)$ & $23(66,67 \%)$ & $153(86,44 \%)$ \\
\hline
\end{tabular}

IMC : Indice de Masse Corporelle ; TT : Tour de Taille.

\section{Discussion}

Les résultats montrent en ce qui concerne l'étude de l'obésité des étudiants, une corpulence normale aussi bien par rapport à l'IMC que par rapport au tour de taille (74,01 \% par rapport à l'IMC, 80,44\% par rapport au TT). En effet, cette population bien active ne connaît pas encore l'aisance socio-économique. Cependant, la prévalence de la surcharge pondérale existe et en plus augmente de manière significative avec l'âge quel que soit le sexe. Dans l'ensemble, il y a 20,51 \% de surpoids et 1,13 \% d'obésité. A notre connaissance, une telle étude n’a pas encore été réalisée dans les universités de Côte d'Ivoire. Cependant, le taux ici est élevé par rapport aux travaux antérieurs de Lokrou réalisés dans 16 lycées publics du district d'Abidjan, qui avaient permis de montrer que $1,42 \%$ étaient en surpoids et $0,9 \%$ obèses (Lokrou and Nioblé C., 2008). Ailleurs, une étude similaire dans une 
université de Douala a également montré une prévalence de surpoids et d'obésité des étudiants presque similaire (19,4\% de surpoids et 3,6\% d'obèses) (Ewane et al, 2012). Cette étude indique aussi que la prévalence de la surcharge pondérale augmente avec l'âge aussi bien chez les hommes que chez les femmes. Cette augmentation de la surcharge pondérale pourrait s'expliquer par une diminution de l'activité physique avec l'âge (Sallis et al., 1993 ; Kimm et al., 2002). Aussi, la prévalence de la surcharge pondérale est plus importante chez les femmes que chez les hommes (26,44 \% vs 1,11 $\%$ pour l'obésité abdominale ; 16,09 \% vs 11,11\% pour l'obésité totale). Cela a aussi été montré par les travaux de Ewane et al. en 2012, et ceux de Kramoh et al. en 2012 ; également dans une étude réalisée sur les élèves du secondaire du district d'Abidjan, d'après une surcharge pondérale évaluée par rapport à l'IMC. Les travaux de l'Institut National Médico-Social (INMES) sur les étudiants au Bénin avaient également indiqués cette prévalence du surpoids et de l'obésité chez les filles (Yessoufou et al., 2012). En effet, les stéroïdes sexuels féminins, notamment les œstrogènes jouent un rôle non négligeable dans la prise de masse grasse (Lovejoy, 1998 ; Mayes et Watson, 2004). Pour ce qui est des antécédents familiaux l’hypertension artérielle est l'antécédent familial le plus fréquent, noté dans $56,50 \%$, des cas ; le diabète est aussi important soit 22,03\% d'étudiants qui ont un parent diabétique. Comme souligné par des travaux effectués en Algérie l'hypertension artérielle et le diabète sont des antécédents familiaux très fréquents (INSP, 2010).

Cette étude a permis de montrer que la majorité des étudiants 74,01 $\%$ ont un poids normal. Il y a cependant 20,51 \% qui sont en surpoids et 1,13 $\%$ qui sont obèses. Cette surcharge pondérale est bien plus fréquente chez les femmes que chez les hommes. En ce qui concerne l’obésité abdominale, elle est également beaucoup plus prépondérante chez les femmes.

\section{Conclusion}

Ce travail a consisté à l'étude de la surcharge pondérale des étudiants. Ce qui nous a permis de montrer que la majorité des étudiants ont un poids normal, il y a cependant certains qui sont en surpoids et d'autres qui sont obèses. Cette surcharge pondérale est bien plus fréquente chez les femmes que chez les hommes. En ce qui concerne l'obésité abdominale, elle est également beaucoup plus prépondérante chez les femmes. Il serait ainsi intéressant de sensibiliser à notre niveau qu'être gros est source de maladie et cela a un effet sur l'espérance de vie.

\section{References :}

1. Ewane M. E., Epacka E. M, Mandengue S. H., Priso B. E., Moumbe T. et Founda B. A. (2012). Dépistage des maladies cardiovasculaires 
chez les étudiants de l'université de Douala et influence des activités physiques et sportives. Pan Afr. Med. J., 11, 77.

2. INSP (Institut National de Santé Publique), 2010. L’Obésité chez l'adulte de 35 à 70 ans en Algérie. Transition épidémiologique et système de santé. Projet TAHIN, P27.

3. Kimm S. Y., Glynn N. W., Kriska A. M., Barton B. A., Kronsberg S. S., Daniels S. R., Crawford P. B., Sabry Z. I. and Liu K. (2002). Decline in physical activity in black girls and white girls during adolescence. N. Engl. J. Med., 347(10), 709-15.

4. Kramoh K. E., Ngoran Y. N. K., Boka B. C., Harding D. B. J. et Guikahué M. K. (2012). Prévalence de l'obésité en milieu scolaire en Côte d'Ivoire. Elsevier Masson, 61(3), 145-149.

5. Lokrou, A. et Nioblé C. (2008). Prévalence du surpoids et de l'obésité en milieu scolaire en Côte d'Ivoire. Elsevier Masson, 2(3), 303-304.

6. Lovejoy J. C. (1998). The influence of sex hormones on obesity across the female life span. Journal of Women's Health,7(10), 12471256.

7. Mayes J. S. et Watson G. H. (2004). Direct effects of sex steroid hormones on adipose tissues and obesity. Obes. Rev, 5(4), 197-216.

8. Olshansky S. J., Passaro D. J., Hershow R. C., Layden J., Carnes B. A., Brody J., Hayflick L., Butler R. N., Allison D. B. and Ludwig D. S. (2005). A potential decline in life expectancy in the United States in the 21st century. N. Engl. J. Med., 352, 1138-45.

9. Organisation mondiale de la santé (OMS) (2003). Obésité: prévention et prise en charge de l'épidémie mondiale. Série de rapports techniques N894, Genève.

10. Sallis J.F. (1993). Epidemiology of physical activity and fitness in children and adolescents. Crit. Rev. Sci. Nutr., 33, 405-408.

11. Yessoufou G., Attakpa E., Baba Moussa F., Hounhouigan J. D., Baba-Moussa L. et Sezan A. (2012). Prévalence et rôle des lipides dans l'avènement de l'obésité au Bénin. Int. J. Biol. Chem. Sci., 6(4), 1695-1702. 